\title{
Echocardiographic Characteristics of Left Ventricular Diastolic Dysfunction among Impaired Glucose Tolerance Patients
}

\author{
AKM Mohiuddin Bhuiyan', Zakia Sultana', Md. Fazlul Kader Khan ${ }^{3}$, Abu Foyez M. Motiour Rahman \\ M Razib Ahsan ${ }^{5}$, Md Mukhlesur Rahmam6, KAM Mahbub Hasan7, M Touhidul Haq ${ }^{8}$ \\ ${ }^{1}$ Junior Consultant, Department of Cardiology, National Institute of Ophthalmology and Hospital, Bangladesh; ${ }^{2}$ Assistant Surgeon, \\ National Institute of Ophthalmology, Dhaka, Bangladesh; ${ }^{3}$ Assistant Professor (Paediatrics), attached in Dept. Of cardiology \\ National Institute of Ophthalmology, Dhaka, Bangladesh; ${ }^{4}$ Assistant Professor, Cumilla Medical College, Cumilla, Bangladesh; \\ ${ }^{5}$ Assistant Professor, Department of Cardiology, Sylhet MAG Osmani Medical College, Sylhet, Bangladesh; ${ }^{6}$ Associate \\ Professor, Department of Cardiology, Bangabandhu Sheikh Mujib Medical University, Dhaka, Bangladesh; ${ }^{7}$ Medical \\ Officer, Department of Cardiology, National Institute of Cardiovascular Diseases, Dhaka, Bangladesh; ${ }^{8}$ Professor and \\ Head, Department of Cardiology, Bangladesh Medical College \& Hospital, Dhaka, Bangladesh
}

[Received: 12 November 2019; Accepted: 2 December 2019; Published: 1 January 2020]

\begin{abstract}
Background: The measurement of left ventricular diastolic dysfunction is important among the impaired glucose tolerance patients. Objectives: The purpose of the present study was to see the echocardiographic characteristics of left ventricular diastolic dysfunction among impaired glucose tolerance patients. Methodology: This cross sectional study was carried out in the Department of Cardiology at Bangabandhu Sheikh Mujib Medical University (BSMMU), Dhaka, Bangladesh from July 2005 to June 2007 for a period of two (02) years. Patients with impaired glucose tolerance (IGT) patients attending Bangladesh Institute of Research and Rehabilitation in Diabetes, Endocrine and Metabolic Disorders (BIRDEM), Dhaka, Bangladesh and fulfilled selection criteria were included in this study as group A. The apparently healthy persons without cardiovascular disease were taken as group B. All the study subjects underwent 2D and M-mode echocardiography for chamber enlargement, ventricular hypertrophy and ventricular systolic function according to recommendation of American Society of Echocardiography. Result: 2D and M-mode echocardiographic characteristics were compared between groups. Among the parameters, LA size in the group A was $31.18 \pm 3.49 \mathrm{~mm}$ and in group B was $25.22 \pm 4.95 \mathrm{~mm}$ which was significantly higher $(\mathrm{p}<0.01)$ in the case group. LA size was also compared among left ventricular diastolic dysfunction (LVDD) present and absent group, which was $(32.76 \pm 4.61 \mathrm{vs} 28.79 \pm 4.11 \mathrm{~mm}, \mathrm{p}<0.001)$ significantly higher in the LVDD present group. Conclusion: In conclusion there is a significant differences of echocardiographic characteristics of left ventricular diastolic dysfunction among impaired glucose tolerance patients. [Journal of National Institute of Neurosciences Bangladesh, 2020;6(1): 38-42]
\end{abstract}

Keywords: Echocardiographic Characteristics; Left Ventricular Diastolic Dysfunction; Impaired Glucose Tolerance

Correspondence: Dr. AKM Mohiuddin Bhuiyan, Junior Consultant, Department of Cardiology, National Institute of Ophthalmology and Hospital, Sher-E-Bangla Nagar, Dhaka-1207, Bangladesh; Mobile: +8801711822986; Email: mbhuiyan14@yahoo.com

Conflict of interest: There is no financial conflict of interest relevant to this paper to disclose.

Funding agency: This research project was not funded by any group or any institution.

Contribution to authors: Bhuiyan AKMM, Sultana Z, Khan MFK contributed from the protocol preparation, data collection up to report writing. Manuscript writing was performed by Rahman AFMM, Ahsan MR, Rahmam MM, Hasan KAMM, Haq MT has revised the manuscript.

How to cite this article: Bhuiyan AKMM, Sultana Z, Khan MFK, Rahman AFMM, Ahsan MR, Rahmam MM, Hasan KAMM, Haq MT. Echocardiographic Characteristics of Left Ventricular Diastolic Dysfunction among Impaired Glucose Tolerance Patients. J Natl Inst Neurosci Bangladesh, 2020;6(1): 38-42

Copyright: (C2020. Bhuiyan et al. Published by Journal of National Institute of Neurosciences Bangladesh. This article is published under the Creative Commons CC BY-NC License (https://creativecommons.org/licenses/by-nc/4.0/). This license permits use, distribution and reproduction in any medium, provided the original work is properly cited, and is not used for commercial purposes.

\section{Introduction}

Left ventricular dysfunction may be due to impairment of its systolic or diastolic function, or both ${ }^{1}$. Systolic dysfunction can be considered as defect in the ability of 
the myofibrils to shorten against a load, thus, the left ventricle (LV) reduces or loses its ability to eject blood into the high-pressure aorta. In diastolic dysfunction the ventricle cannot accept blood at its usual low pressure, ventricular filling is slow, delayed or incomplete unless atrial pressure increases ${ }^{2}$. There is a growing recognition that congestive heart failure (CHF) caused by a predominantly abnormality in diastolic function which is a common cause for significant morbidity and mortality ${ }^{3}$. The impaired glucose tolerance (IGT) patients without IHD, hypertension and other recognized causes of diastolic dysfunction have also shown diastolic dysfunction ${ }^{4}$. A significant number of normotensive, asymptomatic and non-ischaemic, with IGT subject suffering from some extent of diastolic dysfunction. In Bangladesh Khan et $a 1^{5}$ have performed a study on 27,923 cases of glucosuria at Bangladesh Institute of Research and Rehabilitation in Diabetes, Endocrine and Metabolic Disorders (BIRDEM) and have shown that IGT cases have comprised of $6.6 \%$ cases $^{5}$. They have studied retrospectively on 694 cases with IGT registered at BIRDEM with a view to find out the rate of conversion of IGT to overt diabetes in this population. In a five-year prospective study, $23.8 \%$ converted to diabetes mellitus and the average rate of conversion is $4.7 \%$ cases per year ${ }^{6}$.

Epidemiological evidence supports hyperinsulinemia as a marker for coronary artery diseases (CAD) risk, though an aetiologic role has not been demonstrated ${ }^{3}$. Clinical, epidemiological and pathological studies showed the increased occurrence of clinical congestive heart failure (CHF) in impaired glucose tolerance (IGT) subjects attribute to diabetic cardiomyopathy, which take the form of systolic and diastolic left ventricular dysfunction. Left ventricular diastolic dysfunction may represent the first stage of diabetic cardiomyopathy? ${ }^{7}$. Insulin resistance is common in Bangladesh in IGT subjects, but there is few study about LVDD in this population. Therefore, the present study was carried out to find out the frequency of LVDD in IGT subjects and to find out the association of age and sex with LVDD in IGT. Studies have shown that diabetic cardiomyopathy can reverse and arrest the progression by intense diabetic control ${ }^{8-9}$.

This present study was undertaken to see the echocardiographic characteristics of left ventricular diastolic dysfunction among impaired glucose tolerance patients.

\section{Methodology}

This present cross sectional study was carried out in the Department of Cardiology at Bangabandhu Sheikh
Mujib Medical University (BSMMU), Dhaka, Bangladesh from July 2005 to June 2007 for a period of two (02) years. Patients with impaired glucose tolerance (IGT) patients attending Bangladesh Institute of Research and Rehabilitation in Diabetes, Endocrine and Metabolic Disorders (BIRDEM), Dhaka, Bangladesh and fulfilled selection criteria were included in this study. Patients with the age group of 18 to 60 years, fasting blood sugar less than $7 \mathrm{mmol} / \mathrm{L}$ and 2 hours after $75 \mathrm{gm}$ glucose 7.8 to $11.0 \mathrm{mmol} / \mathrm{L}$ with blood pressure of systolic less than $140 \mathrm{mmHg}$ and diastolic less than $90 \mathrm{mmHg}$, sinus rhythm and negative ETT were selected as study population. Any evidence of pericardial, myocardial or endocardial disease, valvular heart disease, congenital heart disease, known or suspected coronary artery disease, diabetes mellitus patients, atrial fibrillation, renal impairment, pregnant women or patients with poor echocardiography window were excluded from this study. The apparently healthy persons without cardiovascular disease were taken as group B. Informed consents were taken from each subject. ECG, chest X-ray, blood sugar (fasting and 2 hours after $75 \mathrm{gm}$ glucose); fasting lipid profile and serum creatinine; urine for microalbumin level were analyzed and findings documented. Exercise tolerance test (ETT) was done in every case and positive cases were excluded. Echocardiography including Doppler with colour flow imaging study were done by cardiologists. Echocardiography for this study was done by Siemens Acuson CV70 and ALOX-A Colour Doppler SSD-1000, Japan, ultrasound imaging system using $2.5 \mathrm{MHz}$ array phased transducer with ECG gating. All the study subjects underwent $2 \mathrm{D}$ and M-mode echocardiography for chamber enlargement, ventricular hypertrophy and ventricular systolic function according to recommendation of American Society of Echocardiography. Careful attention was paid on valvular, congenital, pericardial, myocardial pathology. Special emphasis was given on wall motion abnormality and graded from normal to dyskinetic motion. LV systolic function was recorded. Doppler examination was performed with the study subjects at the left lateral decubitus position. Each valve was evaluated by the pulsed-wave and continuous-wave Doppler echocardiography followed by colour-flow mapping. Main emphasis was given on different Doppler parameters related to LV diastolic function. The apical four-chamber view was used to assess the transmitral flow and pulmonary venous flow parameters. Flow spectral was printed on Polaroid paper with a printer. Each study subjects underwent 
standardized 12-lead electrocardiographic evaluation. Any change in the ST, T-segment was carefully noted. Exercise tolerance test (ETT) was done using Bruce protocol after proper evaluation, excluding contraindications in patients with normal ECG and highly suspicious for IHD. Symptoms of angina and ST-segment changes were carefully evaluated. All relevant information for each individual study subjects were recorded on predesigned data collection sheet. Collected data were compiled and statistical analyses were done using computer based software, Statistical Package for Social Science (SPSS). To arrive at statistical significance, Chi-square test and unpaired Student's ' $t$ ' test were applied. $\mathrm{P}$ value $<0.05$ was taken as minimum level of significance.

\section{Results}

The present prospective study was carried out in the Department of Cardiology, Bangabandhu Sheikh Mujib Medical University (BSMMU), Dhaka. Fifty consecutive cases of IGT patients who attended BIRDEM hospital and fifty non-IGT subjects, who fulfilled the selection criteria were included in this study. Left ventricular diastolic dysfunction (stage 1 only) was present in $26(52.0 \%)$ cases and 5(10.0\%) cases in group A and B respectively. The difference between this two group was statistically highly significant $(\mathrm{p}=0.0001)$ (Table 1).

Table 1: Status of Left Ventricular Diastolic Dysfunction in Groups

\begin{tabular}{lccc}
\hline LVD Dysfunction & Group A & Group B & P value \\
\hline Present & $26(52.0 \%)$ & $5(10.0 \%)$ & \\
Absent & $24(48.0 \%)$ & $45(90.0 \%)$ & 0.0001 \\
Total & $\mathbf{5 0 ( 1 0 0 . 0 \% )}$ & $\mathbf{5 0}(\mathbf{1 0 0 . 0} \%)$ & \\
\hline
\end{tabular}

Chi-square test was performed to see the level of significance

The 2D and M-mode echocardiographic characteristics were compared between group A and group B. Among, the parameters, LA size in the case group was $31.18 \pm 3.49 \mathrm{~mm}$ and in control group was $25.22 \pm 4.95$ $\mathrm{mm}$, which was significantly higher $(\mathrm{P}<0.01)$ in the case group (Table 2).

The 2D and M-mode echocardiographic characteristics were analyzed among the left ventricular diastolic dysfunction present and absent group in case group. LA size $(32.76 \pm 4.61 \mathrm{~mm}$ vs $28.79 \pm 4.11 \mathrm{~mm}, \mathrm{P}<0.001)$ among the left ventricular diastolic dysfunction present group was significantly higher compared to the left ventricular diastolic dysfunction absent group (Table $3)$.
Table 2: 2D and M-mode Echocardiographic Characteristics of the Study Subjects (Mean+SD)

\begin{tabular}{lccc}
\hline Variables & Group A & Group B & P value \\
\hline AO $(\mathrm{mm})$ & $29.26 \pm 3.80$ & $29.52 \pm 2.76$ & $0.696 \mathrm{~ns}$ \\
LA $(\mathrm{mm})$ & $31.18 \pm 3.49$ & $25.22+4.95$ & $0.002 * *$ \\
ACS $(\mathrm{mm})$ & $18.52 \pm 2.31$ & $18.96+1.85$ & $0.585 \mathrm{~ns}$ \\
IVS $(\mathrm{mm})$ & $8.75+1.00$ & $8.69+0.85$ & $0.853 \mathrm{~ns}$ \\
PW (mm) & $9.02 \pm 0.87$ & $9.02+0.88$ & $1.000 \mathrm{~ns}$ \\
LVIDd (mm) & $45.54 \pm 4.74$ & $44.73+6.76$ & $0.604 \mathrm{~ns}$ \\
LVIDs (mm) & $29.96 \pm 4.24$ & $28.24+4.47$ & $0.652 \mathrm{~ns}$ \\
EF $(\%)$ & $65.46 \pm 5.05$ & $65.25+5.27$ & $0.853 \mathrm{~ns}$ \\
\hline
\end{tabular}

$\mathrm{AO}=$ Aortic diameter; $\mathrm{LA}=$ Left atrial diameter; $\mathrm{ACS}=$ Aortic cusp diameter; IVS=Intraventricular, septum thickness; PW=Posterior wall thickness; LVIDd=Left ventricular internal diameter during diastole; LVIDs=Left ventricular internal diameter during systole; $\mathrm{EF}=$ Ejection fraction; Unpaired Student's 't' test was performed to see the level of significance

Table 3: 2D and M-mode Echocardiographic Characteristics of group A with and without Left Ventricular Diastolic Dysfunction (Mean+SD)

\begin{tabular}{lccc}
\hline Variables & \multicolumn{2}{c}{ LVD Dysfunction } & P value \\
\cline { 2 - 3 } & $\begin{array}{c}\text { Present } \\
(\mathbf{n}=\mathbf{2 6})\end{array}$ & $\begin{array}{c}\text { Absent } \\
(\mathbf{n}=\mathbf{2 4})\end{array}$ & \\
\hline AO $(\mathrm{mm})$ & $28.96+1.91$ & $27.42+2.36$ & $0.200 \mathrm{~ns}$ \\
LA (mm) & $32.76+4.61$ & $28.79+4.11$ & 0.0001 \\
ACS (mm) & $18.35+1.56$ & $18.63+1.64$ & $0.827 \mathrm{~ns}$ \\
IVS (mm) & $8.65+0.97$ & $9.24+1.22$ & $0.142 \mathrm{~ns}$ \\
PW (mm) & $8.57+0.95$ & $9.35+0.76$ & $0.411 \mathrm{~ns}$ \\
LVIDd (mm) & $43.23+3.83$ & $44.63+3.85$ & $0.236 \mathrm{~ns}$ \\
LVIDs (mm) & $29.27+2.28$ & $28.46+2.54$ & $0.679 \mathrm{~ns}$ \\
EF (\%) & $43.60 \pm 2.32$ & $65.48 \pm 4.77$ & $0.237 \mathrm{~ns}$ \\
\hline AO $=$ Aottc dian
\end{tabular}

$\mathrm{AO}=$ Aortic diameter; $\mathrm{LA}=$ Left atrial diameter; $\mathrm{ACS}=$ Aortic cusp diameter; IVS=Intraventricular, septum thickness; PW=Posterior wall thickness; LVIDd=Left ventricular internal diameter during diastole; LVIDs=Left ventricular internal diameter during systole; $\mathrm{EF}=$ Ejection fraction; Unpaired Student's ' $\mathrm{t}$ ' test was performed to see the level of significance

\section{Discussion}

The impact of left ventricular diastolic dysfunction (LVDD) on cardiac morbidity and mortality is becoming increasingly understood and it has become apparent that significant proportion of patients presenting with signs and symptoms of congestive heart failure (CHF) have primary diastolic dysfunction ${ }^{7}$. LVDD is the early manifestation of impaired glucose intolerance (IGT), which is most often asymptomatic in early stage ${ }^{8}$.

Accurate noninvasive assessment is crucial to the broad application and understanding of this common 
condition. Echocardiographic parameters have become the backbone of this noninvasive procedure ${ }^{10}$. The increased incidence of LVDD is the impaired glucose tolerance (IGT) patients explained by presence of early diabetic cardiomyopathy ${ }^{11}$. Bajraktaria et $\mathrm{al}^{10}$ found that $55.0 \%$ patients with IGT had LVDD. This study has endeavoured to find out such recommendation. Detection of LVDD at the entry point of IGT and diabetes mellitus is very important regarding the initiation of mode of management as appropriate mode of management might arrest or reverse the process ${ }^{12}$.

In this study, various grades of LVDD have been evaluated in asymptomatic, normotensive, ETTnegative IGT patients and these are compared with non-IGT age and sex matched controlled subjects by Doppler echocardiography. Until now, there has been few data in Bangladesh regarding the LVDD in asymptomatic, normotensive, ETT negative IGT patients by Doppler echocardiography. In this current study, an attempt has been made to compare data of various relevant studies with that from present study.

In this study 2D and M-mode echocardiographic characteristics are compared between groups. Among the parameters LA size in the group A $(31.18+3.49$ $\mathrm{mm})$ is significantly higher $(\mathrm{P}<0.01)$ than group $\mathrm{B}$ $(25.22 \pm 4.95 \mathrm{~mm})$. Higher LA size in IGT population may reflect increased LVEDP.

Furthermore, the 2D and M-mode echocardiographic characteristics are also compared between LVDD present and absent groups in study population. LA size (31.18 \pm 3.49 vs $26.22 \pm 3.95 \mathrm{~mm}, \mathrm{p}<0.001)$ among the left ventricular diastolic dysfunction present group is significantly higher compared to the LVDD absent group.

Karmakar $^{13}$ in his study showed that $56.25 \%$ subjects had left ventricular diastolic dysfunction in type 2 diabetes mellitus patients, all of whom had delayed relaxation type. Zahurul ${ }^{14}$ in his study showed that $55.0 \%$ subjects had LVDD. This study also showed similar to our study. However, Poirier et $\mathrm{a}^{15}$ showed that $60.0 \%$ cases have LVDD, and among them, 32.0\% cases have impaired relaxation and $28.0 \%$ cases have pseudonormal filling pattern. In this study, the authors does not follow the criteria for Doppler parameters of Garcia et $\mathrm{al}^{16}$ which is most updated Doppler parameters for detection of LVDD. The study showed that $28.0 \%$ subjects have pseudonormal filling pattern. However, Cosson and Kevorkian ${ }^{7}$ have showed that pseudonormal filling pattern group have LVEDP $>16$ $\mathrm{mmHg}$ and almost all the subjects with pseudonormal filling pattern group have NYHA $2.8 \pm 0.5$ heart failure.

\section{Conclusion}

In conclusion echocardiographic characteristics of left ventricular diastolic dysfunction are significantly different among impaired glucose tolerance patients. Furthermore, LVDD is present in significant number in IGT cases who are free of clinically detectable heart disease. Therefore, it has been recommended that a large scale study should be conducted for real scenario.

\section{References}

1. Bajraktari G, Koltai MS, Ademaj F, Rexhepaj N, Qirko S, Ndrepepa G, et al. Relationship between insulin resistance and left ventricular diastolic dysfunction in patients with impaired glucose tolerance and type 2 diabetes. Int J Cardiol, 2006;110:206-21

2. Salmasi AM, Frost P, Dancy M. Left ventricular diastolic function in normotensive subjects 2 months myocardial infarction is related to glucose tolerance. Am Heart J, 2005;150:168-174

3. Power AC. 2001. Diabetes mellitus. In: Braunwald E, et al. (eds). Harrison's principle of internal medicine. Vol. 1, 15th ed. pp.2109-2137.

4. O'Connor CM, Gattis WA, Shaw L, Cuffe MS, Califf RM. Clinical characteristics and long-term outcomes of patients with heart failure preserve systolic function. Am J Cardiol 2000;86:863-867.

5. Khan AR, Ahmed T, Mahtab H. Rate of conversion of IGT to diabetes mellitus: a retrospective study. J Diab Assoc Bang 1988:17:10-15

6. Charney P. 2001. Women and coronary artery disease. In: Fuster V, O'Rourke ARW (eds). Hurst's, the heart. 11th ed. McGraw-Hill, New York; pp.689-699.

7. Cosson S, Kevorkian JP. Left ventricular diastolic dysfunction: an early sign of diabetic cardiomyopathy. Diabetes Metab, 2003;29:455-466.

8. Khan ZA, Haque KMHS. Study of left ventricular diastolic dysfunction in type-2 diabetes mellitus with chronic stable engina. Univ Heart J, BSMMU, July 2003.

9. Haque SA. Detection of left ventricular diastolic dysfunction in first acute myocardial infarction by Doppler Echocardiography. MD Thesis, BSMMU, Dhaka. 2001.

10. Bajraktari G, Qirko S, Rexhepaj N. Non-insulin dependent diabetes as an independent predictor of asymptomatic left ventricular diastolic dysfunction. Croat Med J, 2005;46:225-231.

11. American Medical Association. 2001. Executive summary of third report of the National Cholesterol Education Program (NCEP) expert panel on detection, evaluation, and treatment of high blood cholesterol in adults (Adult Treatment Panel III). JAMA, 285:2486-2496.

12. Bajraktari G, Rexhepaj N, Bakalli A, Elezi S. Reduced left ventricular diastolic function in asymptomatic patients with non-insulin-dependent diabetes mellitus. Med AM 2004;58:339-341.

13. Karmakar PK. Detection of left ventricular diastole dysfunction (LVDD) in asymptomatic, normotensive, ETT negative newly detected type-2 diabetic patients by Doppler echocardiography [thesis]. Dhaka: Bangabandhu Sheikh Mujib Medical University, 2004

14. Zahurul MM. Study of left ventricular diastolic dysfunction in type-2 diabetes mellitus with chronic unstable angina [thesis]. Dhaka: Bangabandhu Sheikh Mujib Medical University, 2003

15. Poirier P, Bogaty P, Garneau C, Marois L, Dumesnil J. Diastolic dysfunction (LVDD) in normotensive men with cell-controlled type-2 diabetes: important maneuvers in 
echocardiographic screening for preclinical cardiomyopathy. Diabetes Care, 2001;24:5-10.

16. Garcia MJ, Thomas JD, Klein AL. New Doppler diabetic echocardiographic application for the study of diastolic function. J Am Coll Cardiol, 1998;32:865-875 